\title{
POINT THERMAL TRANSMITTANCE OF RIB INTERSECTIONS IN CONCRETE SANDWICH WALL PANELS
}

\author{
Benedetti M. ${ }^{1}$, Gervasio P. ${ }^{2}$, Luscietti D. ${ }^{3}$, Pilotelli M. ${ }^{3}$, Lezzi A.M. ${ }^{3 *}$ \\ * Author for correspondence \\ ${ }^{1}$ Dipartimento di Ingegneria dell'Informazione, \\ ${ }^{2}$ Dipartimento di Ingegneria Civile, Architettura, Territorio, Ambiente e di Matematica, \\ ${ }^{3}$ Dipartimento di Ingegneria Meccanica ed Industriale, \\ Università degli Studi di Brescia, via Branze 38, 25123 Brescia \\ E-mail: adriano.lezzi@unibs.it
}

\begin{abstract}
Concrete sandwich panels are widely used building elements. They are made by two reinforced concrete wythes separated by a layer of lightweight material: the central layer is inhomogeneous due to the presence of concrete ribs which tie the external wythes and act as thermal bridges. International Standards allow to evaluate the average thermal transmittance of concrete sandwich panels as a linear combination of the transmittance of the solid concrete ribs and of the lightened parts - calculated as if the temperature field were 1D - and linear and point thermal transmittances associated with thermal bridges. In a recent work we have addressed the problem of finding an accurate correlation for prediction of linear thermal transmittance values. The goal was reached upon use of a fast and accurate Spectral Element Method. In this work we complete our study investigating the point thermal bridges and determining the associated point thermal transmittance. Point thermal transmittances in sandwich panels are associated with the concrete rib intersections, like in the four panel corners, and require 3D numerical simulations for their evaluation: the computational effort required to approximate the point transmittance is much larger than that needed to estimate the linear one. For this reason we present and discuss a solution strategy based on the use of low-order polynomials $(p=4)$ on three grids of increasing refinement, starting from a very coarse one: results have been improved through an iterated application of Richardson extrapolation. This procedure assures a good trade-off between accuracy, as required by Standards, and computational cost. A dataset of 1080 point transmittance values is obtained varying systematically six geometrical and thermophysical parameters. A simple power law correlation in terms of a single variable depending on linear transmittance of the intersecting ribs is introduced and its accuracy assessed.
\end{abstract}

\section{INTRODUCTION}

Precast concrete sandwich wall panels allow fast and economical constructions of buildings such as factories, warehouses, and malls. Since they are usually produced far from the construction site, weight becomes a crucial point with regard to handling, transportation, and installation issues. The easiest way to meet both the requirements of structure robustness and weight containment, is to have a frame of concrete and fill the empty zones with lightweight materials like expanded polystyrene. In what follows this kind of panel will be referred to as LSP, precast concrete Lightened Sandwich wall Panel. It is worth underlying that LSPs are not designed to be thermal efficient because the use of insulating slabs is aimed to reduce weight, reduction of the average thermal transmittance is just a by-product effect.

In a LSP two prestressed concrete wythes are separated by a heterogeneous layer made by lightweight slabs and concrete ribs: so there are panel regions made of solid concrete which act as thermal bridges. In principle, computation of the thermal transmittance $U$ of LSPs is not a critical issue: ISO 6946, ISO 14683, and ISO 10211 [1, 2, 3] describe accurate methods to do that. These methods require the knowledge of linear and point thermal transmittance associated with thermal bridges in LSPs. Their values can be computed upon numerical simulations performed as described in ISO 10211. However, most panel manufacturers are SMEs (Small-Medium Enterprises) and their technical staff does not have either the know-how, nor the time to numerically compute values necessary to determine the transmittance $U$ of their product range: they consider much more convenient the usage of transmittance catalogues or correlations, easily implemented in a spreadsheet or in an in-house code.

In a previous paper [4] we addressed the problem of finding an accurate correlation for prediction of linear thermal transmittance values of LSPs. In the present work we complete our study investigating the point thermal bridges in LSPs and determining the associated point thermal transmittance.

Point thermal bridges in LSPs coincide with concrete rib intersections, like in the four panel corners. ISO 14683 states that, in general, the effect of point thermal bridges "insofar they result from the intersection of linear thermal bridges", can be neglected [2, Clause 4.2]. In past years, we had to evaluate in a few real cases point thermal bridge contribution in LSPs and found that it accounted for up to $2 \%$, approximately. Besides, the associ- 


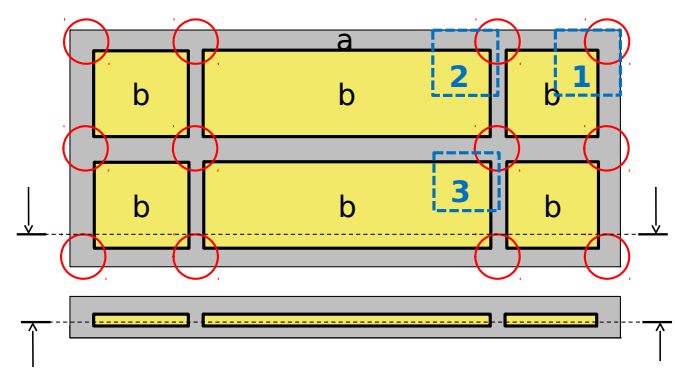

Figure 1. Plan and section view of a LSP: $a$, solid concrete section; $b$, lightened section. Thick lines and open circles indicate linear and point thermal bridges, respectively. Domains 1,2 and 3 are examples of 3D models used in numerical simulations.

ated point transmittances were negative: neglecting them implied overestimating panel transmittance $U$. In this work we check the correctness and the generality of the latter conclusion, on the basis of a systematic study of point transmittance as a function of concrete and lightweight material conductivities and panel geometrical parameters.

Evaluation of point thermal transmittance requires 3D numerical simulations, besides the knowledge of linear transmittance associated with the intersecting concrete ribs. As in [4] we use a conformal quadrilateral Spectral Element Method (SEM): the computational effort required to approximate point transmittances is much larger than that needed to estimate linear ones. That forces us to use coarse meshes and to approximate the temperature field in each mesh element with low-order polynomials, but with the major drawback of loss of accuracy. Here we present and discuss a solution strategy that allows to by-pass this problem. The numerical problem is solved on three grids of increasing refinement upon use of low-order polynomials $(p=4)$ : results are extrapolated by Richardson method $[5,6]$. This procedure assures a good trade-off between accuracy, as required by International Standards, and computational cost.

A dataset of point transmittance values is obtained varying systematically material conductivities and thickness of external and central layers, for the most frequent pairs of rib widths in current panel production. We propose a simple power law correlation in terms of a new variable depending on linear transmittances and concrete wythes thickness. This correlation allows to estimate point transmittances within a relative error of $\pm 10 \%$ which is intermediate between the typical accuracy of numerical calculation of linear thermal transmittance $( \pm 5 \%)$ and that of linear thermal bridge catalogues $( \pm 20 \%)$ [2, Clause 5.1].

To our knowledge, in literature there are only a few other studies on point thermal bridges in precast concrete panels and none of them is concerned with evaluation of point transmittance associated with rib intersections. Studies $[7,8,9]$ do consider point transmittances, but they are concerned with the effect of metal connectors used to tie the concrete wythes in insulated sandwich wall panels.

\section{AVERAGE THERMAL TRANSMITTANCE OF A LIGHT- ENED SANDWICH PANEL}

The average thermal transmittance $U$ of a wall panel is defined as $U=q / A \Delta T$ where $q$ is the heat flow rate through the panel, $\Delta T$ is the temperature difference between the internal and the external environments separated by the panel, and $A$ is the panel area. The four panel edges are considered adiabatic.

Although $q$ could be calculated upon numerical solution of the conduction equation for the entire panel, International Standards $[2,3]$ suggest a more efficient method based on analytical and numerical solutions for a limited number of parts of the panel. Following this approach $U$ is written as,

$$
U=\frac{q}{A \Delta T}=\frac{1}{A}\left(\sum_{i} A_{i} U_{i}+\sum_{j} l_{j} \psi_{j}+\sum_{k} n_{k} \chi_{k}\right)
$$

In Eq. (1) $A_{i}$ and $U_{i}$ are area and thermal transmittance of the $i$-th section of the panel; $l_{j}$ and $\psi_{j}$ are lenght and linear transmittance of the $j$-th linear thermal bridge; $n_{k}$ and $\chi_{k}$ are number and point transmittance of the $k$-th point thermal bridge. Following ISO 6946 [1, Clause 6.2.2], here section denotes a panel part made of thermally homogeneous layers. As clearly shown in Figure 1, a LSP is made of two sections: the solid concrete part, corresponding to ribs (section a); and the three-layer lightened part, made of the concrete wythes and the lightened layer that separates them (section $b$ ).

The thermal transmittance of the two sections, $U_{a}$ and $U_{b}$, is easily calculated in terms of surface resistances, $R_{s e}$ and $R_{s i}$, and of thermal resistances of the homogeneous layers. Therefore, the first sum in Eq. (1), $\sum_{i} A_{i} U_{i}$ represents the transmission heat coefficient through the panel as if the sections were thermally insulated one from the other, and the temperature field were 1D within each section.

The other two sums, $\sum_{j} l_{j} \psi_{j}$ and $\sum_{k} n_{k} \chi_{k}$, represent the corrections associated with linear and point thermal bridges, that is with the regions where the temperature field is $2 \mathrm{D}$ and $3 \mathrm{D}$.

In LSPs, the temperature field is closely approximated by a 2D field near the interfaces between concrete ribs and lightweight slabs (thick lines in Figure 1), whereas it is fully 3D in a neighborhood of the intersections of ribs (open circles in Figure 1).

The linear thermal transmittances $\psi_{j}$ must be evaluated upon solving the conduction equation on 2D domains as described in [4]. Evaluation of the point thermal transmittances $\chi_{k}-$ which is the goal of this study - requires solution of the conduction equation on proper 3D domains which represent panel parts centered around rib intersections. These 3D geometrical models must be identified in accordance with ISO 10211.

\section{POINT TRANSMITTANCE CALCULATION \\ Problem description}

In Figure 2 it is sketched the 3D model used to determine point thermal transmittance values: it represents the part of a panel near the intersection of two ribs. The L-shaped region formed 


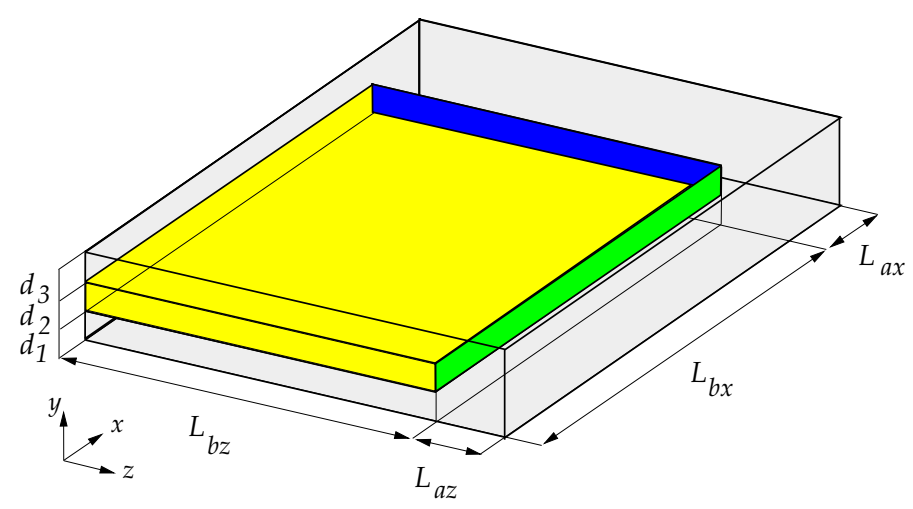

Figure 2. The computational domain $\Omega$, the intersecting ribs form two linear and one point thermal bridges. The linear thermal transmittances are considered as associated with the blue $\left(\psi_{x}\right)$ and green $\left(\psi_{z}\right)$ interfaces.

by the intersecting ribs, represents the solid concrete section $a$, whereas the three-layered region is the lightened section $b$.

The 3D geometrical model coincides with the parallelepiped $\Omega$ of sides $L_{x}=L_{a x}+L_{b x}$ and $L_{z}=L_{a z}+L_{b z}$, and height (panel thickness) $d=d_{1}+d_{2}+d_{3} . d_{1}$ and $d_{3}$ denote the thickness of the external and internal wythe, respectively, whereas $d_{2}$ is the ligthweight material thickness.

On the domain boundaries the following conditions must be satisfied. On the external and internal surfaces $\partial \Omega_{e}(y=0)$ and $\partial \Omega_{i}(y=d)$ Robin condition is imposed. The heat transfer from surface $\partial \Omega_{e}$ to the external environment at temperature $T_{e}$ is characterized by a heat transfer coefficient $h_{e}$ and a surface resistance $R_{s e}=1 / h_{e}$. Internal environment temperature $T_{i}$, heat transfer coefficient $h_{i}$, and surface resistance $R_{s i}=1 / h_{i}$ characterize heat transfer to the internal surface $\partial \Omega_{i}$.

Planes $x=0, x=L_{x}, z=0$, and $z=L_{z}$ are cut-off planes as defined in ISO 10211 [3, Clause 5.2], which coincide with adiabatic surfaces: the union of all adiabatic lateral surfaces will be denoted $\partial \Omega_{a}$.

Boundaries $x=L_{x}$ and $z=L_{z}$ belong to either an adiabatic lateral surface of the panel (as in domains 1 and 2 in Figure 1) or a symmetry plane of an internal rib (as in domains 2 and 3 in Figure 1): therefore $L_{a x}$ and $L_{a z}$ are either the width of a bounding rib or the half-width of an internal rib.

Boundaries $x=0$ and $z=0$ are placed so far from the intersection that the temperature field on them is $2 \mathrm{D}$ for all practical purposes. According to ISO 10211 the distances $L_{b x}$ and $L_{b z}$ between the cut-off planes and the point thermal bridge must be larger or equal to $\max (1 \mathrm{~m}, 3 d)$, where $d$ is the total thickness of the panel.

Denoting $q$ the heat flux through the $3 \mathrm{D}$ domain $\Omega$, Eq. (1) simplifies as follows,

$$
\frac{q}{\Delta T}=A_{a} U_{a}+A_{b} U_{b}+L_{b z} \psi_{x}+L_{b x} \psi_{z}+\chi
$$

where $A_{a}+A_{b}$ is equal to the area of surfaces $\partial \Omega_{e}$ and $\partial \Omega_{i} . \psi_{x}$ and $\psi_{z}$ are the linear transmittances associated with the interfaces between concrete and lightweight material that are highlighted in blue and green, respectively, in Figure 2.

Upon numerical evaluation of $q$ and $\psi_{x}$ and $\psi_{z}$ (see [4]) the point transmittance $\chi$ associated with the rib intersection modelled in Figure 2, can be computed as

$$
\chi=\frac{q}{\Delta T}-\left(A_{a} U_{a}+A_{b} U_{b}+L_{b z} \psi_{x}+L_{b x} \psi_{z}\right)
$$

\section{Input data}

The point transmittance $\chi$ as defined by Eq. (3) depends on several parameters: geometrical, $L_{a x}, L_{a z}, L_{b x}, L_{b z}, d_{1}, d_{2}, d_{3}$; and thermophysical, $\lambda_{c o}, \lambda_{l w}, R_{s e}, R_{s i}$.

In this study $R_{s e}, R_{s i}, L_{b x}$, and $L_{b z}$ are fixed, and $\chi$ is computed for varying values of the other variables. The surface resistances are set equal to the conventional values prescribed by ISO 6946 [1, Clause 5.2]: $R_{s e}=0.04 \mathrm{~m}^{2} \mathrm{~K} / \mathrm{W}$ and $R_{s i}=0.13 \mathrm{~m}^{2} \mathrm{~K} / \mathrm{W}$.

As observed in [4] linear transmittance $\psi$ associated with a concrete rib tends asymptotically to a constant value as the lightweight slab length increases ( $L_{b x}$ and $L_{b z}$ in Figure 2). As a matter of fact the growth is rapid: for length larger than 0.25 $\mathrm{m}$ the value of $\psi$ is hardly distinguishable from the asymptotic value. Since in real panels the lightweight slab length and width are rarely smaller than $0.5 \mathrm{~m}$, the asymptotic value only is needed for all practical purposes. A similar behaviour is expected to characterize the dependence of $\chi$ on $L_{b x}$ and $L_{b z}$ : therefore, following ISO 10211, in all computations we set $L_{b x}=L_{b z}=1 \mathrm{~m}$.

In [4] linear transmittance $\psi$ were computed for about 38,000 different combinations of $\left(L_{a x}, d_{1}, d_{2}, d_{3}, \lambda_{c o}, \lambda_{l w}\right)$. Due to the larger computational cost of 3D simulations, here $\chi$ has been calculated for a subset of that dataset. In particular, only three values for both concrete and lightweight material conductivity have been considered: $\lambda_{c o}=1.6,2.0$, and $2.4 \mathrm{~W} / \mathrm{m} \mathrm{K} ; \lambda_{l w}=0.02$, 0.04 , and $0.06 \mathrm{~W} / \mathrm{m} \mathrm{K}$.

The dependence of point transmittances on solid concrete section widths $L_{a x}$ and $L_{a z}$ is similar to the one on $L_{b x}$ and $L_{b z}$ : it attains its asympotic value for widths larger than $0.2 \mathrm{~m}$, approximately. Here we study the three most frequent values of rib width or half-width: $0.05,0.10$, and $0.20 \mathrm{~m}$. In addition, we compute also the asymptotic cases which can be approximated setting widths equal to $1 \mathrm{~m}$.

W.r.t. Figure 2, for symmetry reasons the value of $\chi$ is invariant when $L_{a x}$ and $L_{a z}$ commute, thus only combinations $L_{a x} \leq L_{a z}$ need to be considered.

In current production of LSPs the concrete wythes have usually the same thickness, therefore analysis has been restricted to the symmetric case $d_{1}=d_{3}$. Three values of wythe thickness have been considered: $0.04,0.06$, and $0.08 \mathrm{~m}$. They are the most frequent for panels of total thickness less than or equal to 0.24 $\mathrm{m}$. Thickness of the lightened layer, $d_{2}$, has been varied among: $0.04,0.06,0.08,0.12$, and $0.16 \mathrm{~m}$. However, the following constraint on panel total thickness has been imposed: $d \leq 0.24 \mathrm{~m}$ : only 12 combinations of values of $d_{1}$ and $d_{2}$ satisfy this condition. 
1080 numerical estimates of $\chi$ have been obtained upon varying $\left(L_{a x}, L_{a z}, d_{1}, d_{2}, \lambda_{c o}, \lambda_{l w}\right)$ as specified above.

\section{NUMERICAL SOLUTION}

The conduction problem described in the previous section is solved through Spectral Element Methods (SEM). SEM are high accurate methods designed to discretize partial differential equations. Their best performance (in terms of computational efficiency) is achieved when the differential problem is set on cartesian geometries, exactly as in the problem we are dealing with, since SEM exploit the tensorial structure of the basis functions. The accuracy of SEM is restricted by the regularity of the data: the thermal conductivity and the prescribed boundary conditions. A brief description of SEM as applied to transmittance computation in LSPs can be find in [4, Sec. 4], whereas for an in-depth description of SEM and of their use to approximate partial differential equations, we refer, e.g., to [10, 11]. Here we present only features and terms necessary to understand the solution strategy adopted.

To approximate the temperature field $T$ inside the panel the computational domain $\Omega$ is partitioned in $N_{e}$ non-overlapping parallelepiped $Q_{k}$ (also named elements) of size $h$ (tipically $h$ denotes the diagonal), such that two adjacent elements share a vertex, an edge or a complete face. Such a partition will be denoted by $Q_{b}=\bigcup_{k=1}^{N_{e}} Q_{k}$. We accept that the elements $Q_{k}$ can have different size $h_{k}$, in such a case we set $h=\max _{k} h_{k}$.

Given a partition $Q_{h}$ of $\Omega$ we look for an approximation $T_{h}$ of $T$ that is globally continuous on $\bar{\Omega}$ and locally (that is in each element $Q_{k}$ ) is a polynomial of degree $p$ with respect to each variable. If the surfaces of discontinuity of the thermal conductivity do not cut any element $Q_{k}$, it can be proved that the discrete solution $T_{h}$ converges to $T$ when the mesh size $h$ tends to zero and/or the polynomial degree $p$ grows to infinity.

Once the discrete temperature $T_{h}$ is available, the heat flux through surfaces $\partial \Omega_{i}$ and $\partial \Omega_{e}$ can be computed by the following formulas:

$$
\begin{aligned}
& q_{i, h}=\int_{\partial \Omega_{i}} \lambda_{c o} \frac{\partial T_{h}(\mathbf{x})}{\partial n} d \partial \Omega=\int_{\partial \Omega_{i}} h_{i}\left(T_{i}-T_{h}\right) d \partial \Omega \\
& q_{e, h}=-\int_{\partial \Omega_{e}} \lambda_{c o} \frac{\partial T_{h}(\mathbf{x})}{\partial n} d \partial \Omega=\int_{\partial \Omega_{e}} h_{e}\left(T_{h}-T_{e}\right) d \partial \Omega .
\end{aligned}
$$

$q_{i, h}$ and $q_{e, h}$ are the discrete approximation of the heat flux through $\Omega, q$, that is required to calculate the point transmittance $\chi$ by Eq. (3). In particular, $q_{i, h} \rightarrow q$ and $q_{e, h} \rightarrow q$ when $h \rightarrow 0$ and/or $p \rightarrow \infty$. The SEM has been implemented in MATLAB.

\section{Discretization strategies}

In order to get a good trade-off between accuracy and computational time one has to choose properly the partition $Q_{b}$ and the polynomial degree $p$.

For each set of data, and any fixed $p$, the discrete fluxes, Eq. (4), have been computed for three different meshes: $Q_{h}$ (named coarse), $Q_{h / 2}$ (medium), and $Q_{h / 4}$ (fine). Therefore, the corresponding point transmittances $\chi_{h, p}, \chi_{h / 2, p}$, and $\chi_{h / 4, p}$ have been evaluated by using Eq. (3), in which $q$ is replaced by its discrete counterpart $q_{h}=\left(\left|q_{i, h}\right|+\left|q_{e, h}\right|\right) / 2$.

Finally, $\chi_{h, p}, \chi_{h / 2, p}$, and $\chi_{h / 4, p}$ are used to better estimate the point transmittance $\chi$ through the Richardson extrapolation technique (see, e.g. [6, Sec. 9.6]), that in our case (with data from three different meshes and the parameter $h$ that is halved at each step) reads

$$
\chi_{e, p}=\frac{8 \chi_{h / 4, p}-6 \chi_{h / 2, p}+\chi_{h, p}}{3} .
$$

In view of the convergence estimate of Richardson extrapolation (see, e.g., [6, Eq. (9.35)]), there exists a positive constant $C$, independent of $h$, but possibily depending on $p$ such that $\left|\chi_{e, p}-\chi\right| \leq C(p)(h / 4)^{3}$. The Richardson extrapolation turns out to be very efficient to our purpose. As a matter of fact, even if the SEM appproximation error vanishes as $h$ when $h \rightarrow 0$ in view of the low regularity of the thermal conductivity, Richardson extrapolation allows to gain third order accuracy w.r.t. $h$ by using a set of three meshes of moderate sizes $h, h / 2$, and $h / 4$, instead of a unique mesh with a very small mesh-size, that would require very large computational effort.

In our simulations we have chosen to use polynomial degree $p=4$. This choice is motivated by several numerical tests, carried out to measure both the computational effort required to solve the linear system associated with the SEM discretization of the conduction problem, and the accuracy of the computed point transmittance.

As test case to study discretization error and computational effort, we have chosen the case: $L_{a x}=L_{a z}=0.05 \mathrm{~m}, d_{1}=d_{3}=$ $0.06 \mathrm{~m}, d_{2}=0.12 \mathrm{~m}, \lambda_{c}=2.0 \mathrm{~W} / \mathrm{mK}, \lambda_{l w}=0.04 \mathrm{~W} / \mathrm{mK}$, and on the associated geometry we have designed the following partitions.

The coarse mesh $Q_{b}$ is obtained by defining $6 \times 4 \times 6(=144)$ elements with side sizes $h_{x}=[0.6,0.2,0.1,0.05,0.05, \mid 0.05]$, $h_{y}=[0.06,|0.06,0.06| 0.06$,$] , and h_{z}=h_{x}$. Vertical pipes highlight where the thermal conductivity is discontinuous. The ratio between the maximum and the minimum size $h_{k}$ is about 10 , with $\max _{k} h_{k} \simeq 0.85$ and $\min _{k} h_{k} \simeq 0.09$. The medium mesh $Q_{h / 2}$ is obtained by halving (along each direction) any element of $Q_{h}$, therefore we have $12 \times 8 \times 12(=1152)$ elements; while the fine mesh $Q_{b / 4}$ is obtained by halving (along each direction) any element of $Q_{h / 2}$, here we have $24 \times 16 \times 24(=9216)$ elements.

The CPU-times (in seconds) needed to compute the temperature field on an Intel i5-3470 4core, $64 \mathrm{bit}, 3.6 \mathrm{GHz}$ and $8 \mathrm{~GB}$ of RAM, are reported in Table 1, left, for $p=2, \ldots, 6$ and the three partitions $Q_{b}, Q_{h / 2}$ and $Q_{b / 4}$. Least square approximation of the measured values provides CPU-time $\simeq 10^{-7} p^{5.6} N_{e}^{1.7} \mathrm{~s}$. We conclude that a large computational effort is required when either moderate or large $p$ is used.

To measure the accuracy of our numerical results, we compute the Richardson extrapolation of the point transmittance $\chi_{e, p}$, for any $p=2,4,6$, as well as the relative error w.r.t. $\chi_{e, 6}$ (our best 


\begin{tabular}{l|lll}
\hline$p$ & coarse & medium & fine \\
\hline 2 & $2.19 \times 10^{-1}$ & $3.06 \times 10^{0}$ & $6.70 \times 10^{1}$ \\
4 & $2.05 \times 10^{0}$ & $4.00 \times 10^{1}$ & $1.44 \times 10^{3}$ \\
6 & $1.27 \times 10^{1}$ & $2.27 \times 10^{2}$ & $9.81 \times 10^{3}$ \\
\hline
\end{tabular}

\begin{tabular}{ccc}
\hline$\chi_{e, p}$ & $e_{p}$ & CPU-time \\
\hline$-1.2792 \times 10^{-2}$ & $1.0401 \times 10^{-2}$ & $7.03 \times 10^{1}$ \\
$-1.2661 \times 10^{-2}$ & $3.8467 \times 10^{-5}$ & $1.48 \times 10^{3}$ \\
$-1.2660 \times 10^{-2}$ & -- & $1.01 \times 10^{4}$ \\
\hline
\end{tabular}

Table 1. At left, CPU-time in seconds needed to compute the temperature by SEM discretization. At right, the Richardson extrapolation of the point transmittances for different values of $p$, the errors w.r.t. the best computed value $\chi_{e, 6}$, and the CPU-times (in s)

estimate), i.e.,

$$
\mathrm{e}_{p}=\frac{\left|\chi_{e, p}-\chi_{e, 6}\right|}{\left|\chi_{e, 6}\right|}
$$

The computed point transmittances $\chi_{e, p}$, the errors (6) and the CPU-times needed to estimate $\chi_{e, p}$ (i.e. the total CPU-time needed to compute the discrete temperature on all three meshes) are shown in Table 1, right. We conclude that the best compromise, obtained by minimizing both the CPU-time and the error is achieved for $p=4$.

\section{RESULTS AND DISCUSSION}

1080 numerical estimates of $\chi$ have been obtained varying six parameters:

$$
\chi=\chi\left(L_{a x}, L_{a z}, d_{1}, d_{2}, \lambda_{c o}, \lambda_{l w}\right) .
$$

The interval spanned by $\chi$ over the set of input data ranges between $-4.38 \times 10^{-2}$ and $-0.48 \times 10^{-2} \mathrm{~W} / \mathrm{K}$ : all values are negative. There is no evidence that the dependence of $\chi$ on some of the variables could be neglected because it is much weaker than others.

At the beginning of this study we planned to develop an artificial neural network (ANN) for prediction of $\chi$, as we did for $\psi$ in [4]. However, in [4] to train an ANN able to model correctly the dependence on $L_{a}$ we had to obtain values of $\psi$ for more than ten $L_{a}$ between 0.05 and $1 \mathrm{~m}$. In this study we could not afford the computational cost of tens of $\left(L_{a x}, L_{a z}\right)$ pairs. Nor it seemed useful to develop an ANN for each pair $\left(L_{a x}, L_{a z}\right)$ investigated. A different approach was followed.

According to ISO 14683 the point thermal bridge studied here can be considered as the intersection of two linear bridges associated with transmittances $\psi_{x}\left(L_{a x}, d_{1}, d_{2}, \lambda_{c o}, \lambda_{l w}\right)$ and $\psi_{z}\left(L_{a z}, d_{1}, d_{2}, \lambda_{c o}, \lambda_{l w}\right)^{1}$. We asked ourselves whether the dependence of $\chi$ on the six variables could be captured to some extent by $\psi_{x}$ and $\psi_{z}$, that is whether $\chi$ depend implicitly on $\left(L_{a x}, L_{a z}, d_{1}, d_{2}, \lambda_{c o}, \lambda_{l w}\right)$ through $\psi_{x}$ and $\psi_{z}$ :

$$
\chi=g\left(\psi_{x}, \psi_{z}\right)
$$

\footnotetext{
${ }^{1}$ Variable $d_{3}$ is not listed since we are considering only the case $d_{3}=d_{1}$.
}

For simmetry reason in Eq. (8) $\psi_{x}$ and $\psi_{z}$ must commute, that is $g$ has to depend on commutative functions of $\psi_{x}$ and $\psi_{z}$, such as $\psi_{x} \psi_{z}, \psi_{x}+\psi_{z}$, etc.

As a matter of fact, if computed values of $\chi$ are plotted versus $\psi_{x} \psi_{z}$ data tend to fall within a smooth narrow region of increasing width for increasing $\psi_{x} \psi_{z}$. Data dispersion depends on the original variables, but dependence on $d_{1}$ seems stronger. After a few trials we came up with the following new variable

$$
\xi=\psi_{x} \psi_{z} \sqrt{2 d_{1}}
$$

for which dispersion is substantially reduced (see Figure 3). In Eq. (9) the factor 2 multiplying $d_{1}$ has been introduced because we believe that for the more general case $d_{3} \neq d_{1}$, variable $\xi$ should be defined as $\xi=\psi_{x} \psi_{z} \sqrt{d_{1}+d_{3}}$.

Upon fitting data with a power law, the following correlation for point transmittance has been obtained:

$$
\chi_{c}(\xi)=-0.4391 \xi^{0.7055}
$$

where all quantities are in SI units.

For all practical purposes Eq. (10) supplies a rather good estimate of $\chi$ when the linear transmittances associated with the intersecting ribs are known. In Figure 4 the point transmittance estimated by correlation (10), $\chi_{c}$, is plotted versus the computed value $\chi$ : $97 \%$ of estimates fall within $\pm 10 \%$ band.

Eq. (10) on average neither overpredict nor underpredict significantly $\chi$, since the mean relative deviation, MRD, is equal to $0.09 \%$. Dispersion of predicted values is limited since the standard deviation, $\mathrm{SD}$, is equal to $4.5 \%$. Here, $\mathrm{SD}$ is defined in terms of relative deviation $\mathrm{RD}=\left(\chi_{c}-\chi\right) / \chi$ :

$$
\mathrm{SD}=\sqrt{\frac{1}{N-1} \sum_{i}\left(\mathrm{RD}_{i}-\mathrm{MRD}\right)^{2}}
$$

As mentioned in the Introduction the relative contribution of point thermal bridges to the average panel transmittance $U$ (term $\sum_{k} n_{k} \chi_{k}$ in Eq. (1)) is up to $2 \%$, approximately. Therefore the error introduced upon use of correlation (10) is up to $0.2 \%$ of $U$.

\section{CONCLUSIONS}

This paper deals with the problem of determining point thermal transmittance associated with rib intersections in LSPs. To- 


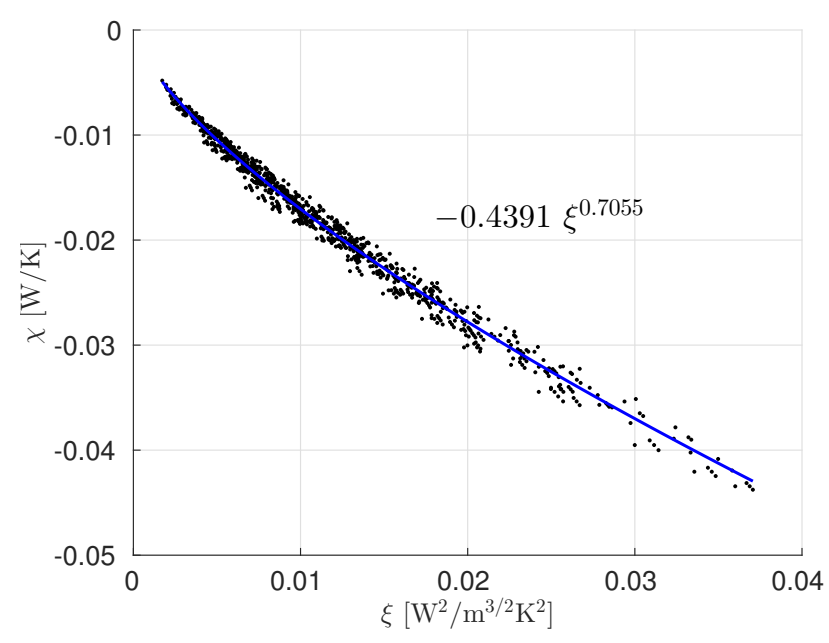

Figure 3. All computed values of $\chi$ vs. the variable $\xi=$ $\psi_{x} \psi_{z} \sqrt{2 d_{1}}$. Solid line: least square approximation of data through a power law

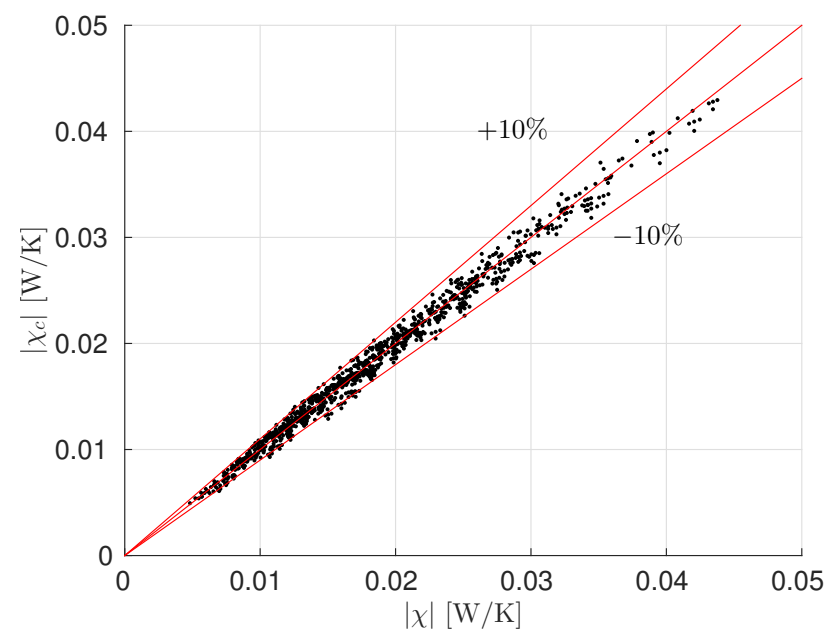

Figure 4. Comparison between correlation predictions, $\left|\chi_{c}\right|$, and computed data $\chi$ : $97 \%$ of estimates fall within $\pm 10 \%$ band

gether with results presented in [4] it allows accurate calculation - within $\pm 1 \%$ - of nominal average thermal transmittance of LSPs according to current International Standards [1, 2, 3].

To reach this goal a dataset of point thermal transmittance associated with rib intersections of LSPs has been built through numerical simulations. 1080 data have been obtained as a function of six parameters: rib widths, thickness of the concrete wythes and of the internal layer, concrete and lightweight material conductivity. The parameters span a range of values typical of current LSPs production. In general, ISO 14683 allows to omit point thermal bridge contribution to LSPs transmittance. For the input data investigated here it is shown that this contribution is always negative: one stays on the safe side neglecting it when evaluating thermal performance of LSPs. Besides, point transmittance values are rather small, their order of magnitude being $10^{-2} \mathrm{~W} / \mathrm{K}$.

Accurate calculation of such a small quantity through numerical solution of heat conduction equation in a 3D domain has been tricky and required a solution strategy based on Richardson extrapolation. This procedure allowed to determine point transmittance values with a relative error which is about $10^{-4}$.

Finally, an explicit correlation is proposed for prediction of point transmittance. Although data show a significant dependence of $\chi$ on each of the six variables mentioned above, we manage to find a simple power law correlation which allows to calculate $\chi$ as a function of a single variable, $\xi=\psi_{x} \psi_{z} \sqrt{2 d_{1}}$. The correlation has standard deviation equal to $4.5 \%$ and predicts more than $97 \%$ of computed data within $\pm 10 \%$. It represents a good practical tool, easily implemented in a spreadsheet or in an in-house code for calculation of $U$.

\section{REFERENCES}

[1] ISO 6946. Building components and building elements Thermal resistance and thermal transmittance - Calculation method. 2007.

[2] ISO 14683. Thermal bridges in building construction - Linear thermal transmittance - Simplified methods and default values. 2007.

[3] ISO 10211. Thermal bridges in building construction Heat flows and surface temperatures - Detailed calculations. 2007.

[4] Luscietti D., Gervasio P., and Lezzi A.M. J. Phys.: Conf. Series, 547:012014, 2014.

[5] Roy C.J. Review of discretization error estimators in scientific computing. 48th AIAA Aerospace Sciences Meeting, pages AIAA 2010-126, 2010.

[6] Quarteroni A., Sacco R., and Saleri F. Numerical Mathematics. 2nd edition. Texts in Applied Mathematics. Springer-Verlag, Berlin, 2007.

[7] Lee B.-J. and Pessiki S. PCI J., 53:86-100, 2008.

[8] Willems W. and Hellinger G. Bauphysik, 32:275-287, 2010.

[9] Kim Y.J. and Allard A. Energy Buildings, 48:137-148, 2014.

[10] Canuto C., Hussaini M.H., Quarteroni A., and Zang T.A. Spectral Methods. Evolution to Complex Geometries and Applications to Fluid Dynamics. Springer, Heidelberg, 2007.

[11] Quarteroni A. Numerical Models for Differential Problems. Springer, Heidelberg, 2014. 